PROCEEDINGS OF THE

AMERICAN MATHEMATICAL SOCIETY

Volume 132, Number 6 , Pages 1621-1625

S 0002-9939(03)07262-9

Article electronically published on December 5, 2003

\title{
ON LINEAR TRANSFORMATIONS PRESERVING AT LEAST ONE EIGENVALUE
}

\author{
S. AKBARI AND M. ARYAPOOR \\ (Communicated by Joseph A. Ball)
}

\begin{abstract}
Let $F$ be an algebraically closed field and $T: M_{n}(F) \longrightarrow M_{n}(F)$ be a linear transformation. In this paper we show that if $T$ preserves at least one eigenvalue of each matrix, then $T$ preserves all eigenvalues of each matrix. Moreover, for any infinite field $F$ (not necessarily algebraically closed) we prove that if $T: M_{n}(F) \longrightarrow M_{n}(F)$ is a linear transformation and for any $A \in M_{n}(F)$ with at least an eigenvalue in $F, A$ and $T(A)$ have at least one common eigenvalue in $F$, then $T$ preserves the characteristic polynomial.
\end{abstract}

\section{INTRODUCTION}

An active research topic in matrix theory is the linear preserver problems that deal with the characterization of linear transformations on matrix spaces with some special properties such as preserving certain functions or subsets. The classification of preserver problems began about 100 years ago. Characterizations of preservers have been appearing regularly over the past thirty years, and an excellent summary of nearly all characterizations of linear preservers can be found in a special issue of linear and multilinear algebra [5]. In 1897, Frobenius [2] proved that for any linear transformation on $M_{n}(\mathbb{C})$ that preserves the determinant, there are two matrices $A$ and $B$ in $M_{n}(\mathbb{C})(\operatorname{det}(A B)=1)$, such that either $T(X)=A X B$ or $T(X)=A X^{t} B$, for any $X \in M_{n}(\mathbb{C})$, where $X^{t}$ denotes the transpose of $X$.

In 1959 Marcus and Moyls [3] extended this result to any arbitrary field and proved the following somewhat surprising theorem.

Theorem A. If $F$ is a field and $T$ is a determinant preserving linear transformations, then there exist matrices $A$ and $B \in M_{n}(F)(\operatorname{det}(A B)=1)$, such that either $T(X)=A X B$ or $T(X)=A X^{t} B$, for any $X \in M_{n}(F)$.

Also, the interesting theorem was proved by Marcus and Purves [4].

Theorem B. Let $F$ be an algebraically closed field and $T: M_{n}(F) \longrightarrow M_{n}(F)$ be a linear transformation. If for any $A \in M_{n}(F), T$ preserves the set of eigenvalues of $A$ (with multiplicity), then there exists an invertible matrix $P \in M_{n}(F)$ such that for any $X \in M_{n}(F)$, either $T(X)=P X P^{-1}$ or $T(X)=P X^{t} P^{-1}$.

Received by the editors December 17, 2002 and, in revised form, February 27, 2003.

2000 Mathematics Subject Classification. Primary 15A04, 47B49.

Key words and phrases. Linear transformation, preserve, eigenvalue. 
Results. In the present paper we want to generalize Theorem A and Theorem B. More precisely we show that for any algebraically closed field $F$, if $T: M_{n}(F) \longrightarrow$ $M_{n}(F)$ is a linear transformation and for any $A \in M_{n}(F), T$ preserves at least one eigenvalue of $A$, then there exists an invertible matrix $P \in M_{n}(F)$ such that either $T(X)=P X P^{-1}$ or $T(X)=P X^{t} P^{-1}$.

Also, for any infinite field $F$, we show that if $T: M_{n}(F) \longrightarrow M_{n}(F)$ is a linear transformation and for any $A \in M_{n}(F)$ with at least an eigenvalue in $F, A$ and $T(A)$ have at least one common eigenvalue in $F$, then there exists an invertible matrix $Q \in M_{n}(F)$ such that for any $X \in M_{n}(F)$, either $T(X)=Q X Q^{-1}$ or $T(X)=Q X^{t} Q^{-1}$.

Before proving our main theorem we need the following key lemma.

Lemma 1. Let $F$ be an infinite field and $A \in M_{n}(F)$. Then there exists a matrix $B \in M_{n}(F)$ such that for infinitely many scalars $x \in F, A+x B$ has at least one eigenvalue in $F$.

Proof. Without loss of generality, we may assume that $A$ is a rational canonical form. Suppose that the rational canonical form of $A$ has $k$ blocks say, $A_{1}, \ldots, A_{k}$. If $A_{i} \in M_{n_{i}}(F)$ is as follows,

$$
A_{i}=\left(\begin{array}{ccccc}
0 & 0 & 0 & \ldots & -a_{i 0} \\
1 & 0 & 0 & \cdots & -a_{i 1} \\
0 & 1 & \ddots & 0 & -a_{i 2} \\
\vdots & \vdots & \ddots & 0 & \vdots \\
0 & 0 & \vdots & 1 & -a_{i, n_{i}-1}
\end{array}\right)
$$

then the characteristic polynomial of $A_{i}$ is $f_{i}(x)=x^{n_{i}}+a_{i, n_{i}-1} x^{n_{i}-1}+\cdots+a_{i 0} \in$ $F[x]$. Since $F$ is infinite, then the image of the function $f_{1}: F \longrightarrow F$ is infinite. Suppose that $B$ is an $n$ by $n$ matrix over $F$, whose $\left(1, n_{1}\right)$-th entry is 1 and the other entries are 0 . For any $r \in \operatorname{Im} f_{1}$, the characteristic polynomial of $A+r B$ is equal to $\left(f_{1}(x)-r\right) \prod_{i=2}^{k} f_{i}(x)$, and therefore $A+r B$ has at least one eigenvalue in $F$.

In the next result, we will use the following well-known simple facts (see Corollary 8.22 and Theorem 8.31 of [1]).

Theorem C. Let $R$ be a unique factorization domain. If $f(t)$ and $g(t)$ are relatively prime polynomials in $R[t]$, then there exist polynomials $p(t), q(t) \in R[t]$ such that $0 \neq f(t) p(t)+g(t) q(t) \in R$.

Theorem D ([6] p. 369, Exercise 3]). If $F$ is a field and $R=F\left[x_{11}, \ldots, x_{n n}\right]$, then $f(t)=\operatorname{det}\left(t I-\left[x_{i j}\right]\right)$ is an irreducible polynomial in $R[t]$.

For any polynomial $f\left(x_{11}, \ldots, x_{n n}\right) \in F\left[x_{11}, \ldots, x_{n n}\right]$ and for each matrix $A=$ $\left[a_{i j}\right] \in M_{n}(F)$, we define $f(A)=f\left(a_{11}, \ldots, a_{n n}\right)$. Now we are in a position to prove our main theorem.

Remark 1. If $F$ is an infinite field and $S$ is an infinite subset of $F$, and furthermore, $f\left(x_{1}, \ldots, x_{n}\right) \in F\left[x_{1}, \ldots, x_{n}\right]$ is a polynomial such that for every $a_{1}, \ldots, a_{n} \in S$, $f\left(a_{1}, \ldots, a_{n}\right)=0$, then $f \equiv 0$. The proof of this fact is based on applying induction on $n$. 
Theorem 1. Let $F$ be an infinite field and $T: M_{n}(F) \longrightarrow M_{n}(F)$ be a function defined as $T\left(\left[x_{i j}\right]\right)=\left[f_{i j}\left(x_{11}, \ldots, x_{n n}\right)\right]$, where $f_{i j}\left(x_{11}, \ldots, x_{n n}\right) \in F\left[x_{11}, \ldots, x_{n n}\right]$. If for any $A \in M_{n}(F)$ with at least an eigenvalue in $F, A$ and $T(A)$ have at least one common eigenvalue in $F$, then $T$ is a characteristic polynomial preserver.

Proof. Let $R=F\left[x_{11}, \ldots, x_{n n}\right]$ and $f(t)$ and $g(t)$ be the following polynomials in $R[t]:$

$$
f(t):=\operatorname{det}\left(t I-\left[x_{i j}\right]\right), \quad g(t):=\operatorname{det}\left(t I-T\left(\left[x_{i j}\right]\right)\right) .
$$

We claim that $f(t) \mid g(t)$ in $R[t]$. If $f(t) \nmid g(t)$, since $R$ is a unique factorization domain and $f(t)$ is an irreducible polynomial (see Theorem B), by Theorem $\mathrm{C}$ there exist polynomials $p(t), q(t) \in R[t]$ such that $0 \neq f(t) p(t)+g(t) q(t) \in R$. Let $\gamma\left(x_{11}, \ldots, x_{n n}\right)=f(t) p(t)+g(t) q(t)$.

We prove that $\gamma \equiv 0$, and thus we have $f(t) \mid g(t)$. Assume that $A=\left[a_{i j}\right] \in$ $M_{n}(F)$. By Lemma 1, there exists a matrix $B \in M_{n}(F)$ such that for infinitely many scalars $x \in F, A+x B$ has an eigenvalue $c_{x} \in F$ that is preserved by $T$. For such values $x$, if $A+x B=\left[g_{i j}(x)\right]$, we have

$$
\left.f\left(c_{x}\right)\right|_{\left[g_{i j}(x)\right]}=\left.g\left(c_{x}\right)\right|_{\left[g_{i j}(x)\right]}=0 .
$$

Therefore, for infinitely many scalars $x \in F$ we have $\gamma\left(\left[g_{i j}(x)\right]\right)=0$. But $\gamma\left(\left[g_{i j}(x)\right]\right)$ is a polynomial in $F[x]$, and it should be the zero polynomial. Now since $A=$ $\left[g_{i j}(0)\right]$, we have $\gamma(A)=0$. Thus by Remark $1, \gamma \equiv 0$ and the claim is proved. Thus we have $f(t) \mid g(t)$. Since $f(t)$ and $g(t)$ are monic and have the same degree in $t$, we have $f(t)=g(t)$. This implies that $T$ preserves the characteristic polynomial.

Now by Theorem 1 and Theorem B we have the following theorem.

Theorem 2. Let $F$ be an algebraically closed field and $T: M_{n}(F) \longrightarrow M_{n}(F)$ be a linear transformation. If $T$ preserves at least one eigenvalue of each matrix, then $T$ preserves all eigenvalues of each matrix. Moreover, there exists an invertible matrix $P \in M_{n}(F)$ such that for any $X \in M_{n}(F)$, either $T(X)=P X P^{-1}$ or $T(X)=P X^{t} P^{-1}$.

Remark 2. We note that the assertion of Theorem 1 is not valid whenever $F$ is finite. To see this define

$$
T: M_{2}\left(\mathbb{Z}_{2}\right) \longrightarrow M_{2}\left(\mathbb{Z}_{2}\right), T\left(\left[\begin{array}{ll}
a & b \\
c & d
\end{array}\right]\right)=\left[\begin{array}{cc}
b+d & a+d \\
c & d
\end{array}\right] .
$$

It is not hard to see that $T$ is a linear transformation with the desired properties, but $T\left(\left[\begin{array}{ll}1 & 0 \\ 0 & 0\end{array}\right]\right)=\left[\begin{array}{ll}0 & 1 \\ 0 & 0\end{array}\right]$ and the characteristic polynomials of $\left[\begin{array}{ll}1 & 0 \\ 0 & 0\end{array}\right]$ and $\left[\begin{array}{ll}0 & 1 \\ 0 & 0\end{array}\right]$ are different.

The following corollary shows that, when $F$ is an algebraically closed field we can reduce the assumption of Theorem 1 .

Corollary 1. Let $F$ be an algebraically closed field and $T: M_{n}(F) \longrightarrow M_{n}(F)$ be a function defined as $T\left(\left[x_{i j}\right]\right)=\left[f_{i j}\left(x_{11}, \ldots, x_{n n}\right)\right]$, where $f_{i j}\left(x_{11}, \ldots, x_{n n}\right) \in$ $F\left[x_{11}, \ldots, x_{n n}\right]$. Let $S \subset F$ be an infinite set. If for every $B \in M_{n}(S), T$ preserves at least one eigenvalue of $B$, then for every $A \in M_{n}(F)$, T preserves all eigenvalues of $A$. 
Proof. By the method used in the proof of Theorem 1 and using Remark 1, the proof is complete.

Lemma 2. Let $F$ be an infinite field. If $f\left(x_{11}, \ldots, x_{n n}\right) \in F\left[x_{11}, \ldots, x_{n n}\right]$ is a polynomial such that for every $A \in G L_{n}(F), f(A)=0$, then $f \equiv 0$.

Proof. For every $A \in M_{n}(F)$, we have $f(A) \operatorname{det}(A)=0$. Now since $F\left[x_{11}, \ldots, x_{n n}\right]$ is a domain and $\operatorname{det}\left(\left[x_{i j}\right]\right)$ is a nonzero polynomial, by Remark 1 we conclude that $f \equiv 0$.

Corollary 2. Let $F$ be an algebraically closed field and $T: M_{n}(F) \longrightarrow M_{n}(F)$ be a function defined as $T\left(\left[x_{i j}\right]\right)=\left[f_{i j}\left(x_{11}, \ldots, x_{n n}\right)\right]$, where $f_{i j}\left(x_{11}, \ldots, x_{n n}\right) \in$ $F\left[x_{11}, \ldots, x_{n n}\right]$. If for every $A \in G L_{n}(F), T$ preserves at least one eigenvalue of $A$, then for every $A \in M_{n}(F), T$ preserves all eigenvalues of $A$.

Proof. By the methods used in the proofs of Theorem 1 and Lemma 2, the proof is complete.

Corollary 3. Let $T: M_{n}(\mathbb{C}) \longrightarrow M_{n}(\mathbb{C})$ be a function defined as $T\left(\left[x_{i j}\right]\right)=$ $\left[f_{i j}\left(x_{11}, \ldots, x_{n n}\right)\right]$, where $f_{i j}\left(x_{11}, \ldots, x_{n n}\right) \in \mathbb{C}\left[x_{11}, \ldots, x_{n n}\right]$. If for any Hermitian matrix $H \in M_{n}(\mathbb{C})$, $T$ preserves at least one eigenvalue of $H$, then for any $A \in$ $M_{n}(\mathbb{C}), T$ preserves all eigenvalues of $A$.

Proof. Assume that $f(t)$ and $g(t)$ and $\gamma\left(x_{11}, \ldots, x_{n n}\right)$ are those polynomials given in the proof of Theorem 1. It is easily seen that each matrix $A \in M_{n}(\mathbb{C})$ can be written as $H_{1}+i H_{2}$, where $H_{1}$ and $H_{2}$ are Hermitian matrices. For any $r \in \mathbb{R}$, since $H_{1}+r H_{2}$ is a Hermitian matrix, as we saw in the proof of Theorem 1, we conclude that $\gamma\left(H_{1}+r H_{2}\right)=0$. On the other hand, $\gamma\left(H_{1}+x H_{2}\right)$ is a polynomial over complex numbers with infinitely many roots and it should be zero. In particular, $\gamma(A)=\gamma\left(H_{1}+i H_{2}\right)=0$. Therefore, we have $f(t) \mid g(t)$. Since $f(t)$ and $g(t)$ are monic and have the same degree in $t$, we have $f(t)=g(t)$. This implies that $T$ preserves all eigenvalues of $A$.

Theorem 3. Let $F$ be an infinite field and $T: M_{n}(F) \longrightarrow M_{n}(F)$ be a linear transformation. If for any $A \in M_{n}(F)$ with at least an eigenvalue in $F, A$ and $T(A)$ have at least one common eigenvalue in $F$, then there exists an invertible matrix $Q \in M_{n}(F)$ such that for any $X \in M_{n}(F)$, either $T(X)=Q X Q^{-1}$ or $T(X)=Q X^{t} Q^{-1}$.

Proof. Consider $T \otimes 1: M_{n}(\bar{F}) \longrightarrow M_{n}(\bar{F})$ as a linear transformation over $\bar{F}$, where $\bar{F}$ is the algebraic closure of $F$ (note that $M_{n}(\bar{F}) \simeq M_{n}(F) \otimes_{F} \bar{F}$ ). By Theorem 1, $T$ is a characteristic polynomial preserver and if in Corollary 1, we put $S=F$, we conclude that for any $A \in M_{n}(\bar{F}), T \otimes 1$ preserves all eigenvalues of $A$. Now by Theorem $\mathrm{B}$, there exists an invertible matrix $P \in M_{n}(\bar{F})$ such that for any $X \in M_{n}(\bar{F})$, either $(T \otimes 1)(X)=P X P^{-1}$ or $(T \otimes 1)(X)=P X^{t} P^{-1}$. We note that the restriction of $T \otimes 1$ to $M_{n}(F)$ is $T$, and this implies that for any $X \in M_{n}(F)$ either $T(X)=P X P^{-1}$ or $T(X)=P X^{t} P^{-1}$. But it is well known that there exists an invertible matrix $Q \in M_{n}(F)$ such that for any $X \in M_{n}(F)$, either $T(X)=Q X Q^{-1}$ or $T(X)=P X^{t} P^{-1}$ and the proof is complete. 


\section{ACKNOWLEDGMENTS}

The first author is indebted to the Research Council of Sharif University of Technology for support. Also, the authors wish to thank the referee for her/his useful comments.

\section{REFERENCES}

[1] Brown, C. W., Matrices over Commutative Rings, Monographs and Textbooks in Pure and Applied Mathematics, Vol. 169, Marcel Dekker, New York, 1993. MR 93k:15028

[2] Frobenius, G., Uber die Darstellung der endlichen Gruppen durch linear Substitutionen, Sitzungsber Deutsch. Akad. Wiss. Berlin, 1897, 994-1015.

[3] Marcus, M. and Moyls, B., Transformations on Tensor Product Spaces, Pacific J. Math. 9 (1959), 1215-1221. MR 21:7219

[4] Marcus, M. and Purves, R., Linear Transformations on Algebras of Matrices II : The Invariance of the Elementary Symmetric Functions, Canad J. Math. 11 (1959), 383-396. MR 21:4167

[5] Pierce, S., et al., A Survey of Linear Preserver Problems, Linear and Multilinear Algebra 33 (1992), 1-129.

[6] Pierce, R. S., Associative Algebras, Graduate Texts in Mathematics 88, Springer-Verlag, New York, 1982. MR 84c:16001

Department of Mathematical Sciences, Sharif University of Technology, P. O. Box 11365-9415, TeHran, Iran

E-mail address: s_akbari@sina.sharif.ac.ir

Department of Mathematical Sciences, Sharif University of Technology, P. O. Box 11365-9415, TEHRAN, IRAN

E-mail address: aryapoor2002@yahoo.com 